\title{
Variable computed tomography appearances of COVID-19
}

\author{
Zhan Ye $\underline{\mathrm{Lim}}^{1}$, MD, Hau Wei Khoo ${ }^{1}$, MD, FRCR, Terrence Chi Hong $\underline{H u i}^{1}$, MBBS, FRCR, Shawn Shi Xian Kok ${ }^{2}$, MBBS, FRCR, \\ Kenneth Eng Ling Kwan ${ }^{1}$, MBBS, FRCR, Barnaby Edward Young ${ }^{3,4}$, MB BChir, MRCP, Cher Heng $\underline{\text { Tan }}^{1,5}$, MBBS, FRCR,
}

Gregory Jon Leng $\underline{K a w}^{1}$, MBBS, FRCR

The coronavirus disease 2019 (COVID-19) is typically diagnosed by specific assays that detect viral nucleic acid from the upper respiratory tract; however, this may miss infections involving only the lower airways. Computed tomography (CT) has been described as a diagnostic modality in the COVID-19 diagnosis and treatment plan. We present a case series with virologically confirmed COVID-19 pneumonia. Variable CT features were observed: consolidation with ground-glass opacities, ground-glass opacities with subpleural reticular bands, and an anterior-posterior gradient of lung abnormalities resembling that of acute respiratory distress syndrome. Evolution of CT findings was observed in one patient, where there was interval resolution of bilateral lung consolidation with development of bronchiolectasis and subpleural fibrotic bands. While sensitive for detecting lung parenchymal abnormalities in COVID-19 pneumonia, the use of CT for initial diagnosis is discouraged and should be reserved for specific clinical indications. Interpretation of chest CT findings should be correlated with duration of symptoms to better determine the disease stage and aid in patient management.

Keywords: computed tomography, coronavirus disease, COVID-19

\section{INTRODUCTION}

Reports in December 2019 from Wuhan, Hubei Province, China, of a cluster of patients with severe pneumonia heralded a pandemic that has affected most of the world. As of 31 March 2020, 750,890 confirmed severe acute respiratory syndrome coronavirus 2 (SARS-CoV-2) cases and 36,405 deaths have been reported globally. ${ }^{(1)}$ While extraordinary measures have been taken by many governments in an effort to slow the spread of this virus, over the long term, control of this outbreak will rely on accurate diagnosis and isolation of infected individuals.

Typically, diagnosis of the novel coronavirus disease 2019 (COVID-19) relies on polymerase chain reaction (PCR) of respiratory specimens to detect SARS-CoV-2 nucleic acid. This test is highly specific, but swabs of the upper respiratory tract may lack sensitivity when the infection is confined to the lower airways. Chest computed tomography (CT) has been shown to have a high sensitivity in the Chinese population. ${ }^{(2)}$ The use of chest CT in the diagnosis of COVID-19 was described in February 2020, in the diagnosis and treatment plan for COVID-19 pneumonia issued by the National Health Commission of the People's Republic of China. ${ }^{(3)}$

We present a case series of three patients with confirmed COVID-19 pneumonia in our institution, followed by a discussion of the role of chest CT for this novel respiratory tract infection. Informed consent from the patients in this study was waived by the Singapore Ministry of Health, under the Infectious Diseases Act (Chapter 137).

\section{CASE SERIES}

\section{Case 1}

A 52-year-old woman with a background of hypertension presented with cough, shortness of breath and fever for four days.
She had no recent travel or contact history. She was admitted for isolation and further management. She was haemodynamically stable and physical examination was unremarkable. Laboratory results revealed mildly elevated C-reactive protein (CRP) of $7.1 \mathrm{mg} / \mathrm{L}(0.0-5.0 \mathrm{mg} / \mathrm{L})$, while neutrophil level was mildly reduced at $1.43 \times 10^{9} / \mathrm{L}\left(1.9-6.60 \times 10^{9} / \mathrm{L}\right)$. Nasopharyngeal reverse transcription-polymerase chain reaction (RT-PCR) tested positive for SARS-CoV-2. Initial chest radiograph revealed right lower zone consolidation and a faint ground-glass opacity in the right upper zone (Fig. 1). During the admission, a CT study of the abdomen and pelvis was performed for evaluation of left flank pain, which revealed left renal calculi. Limited sections of the lung bases demonstrated consolidation with surrounding groundglass opacities in the right lower lobe (Fig. 1). Focal consolidation, seen in the lingula, was not evident on chest radiograph. This illustrates the sensitivity of CT in detecting ground-glass opacities. She remained stable during admission without requiring oxygen supplementation and was discharged well after 11 days.

\section{Case 2}

A 56-year-old man from Shandong, China, presented with fever and cough for a week, as well as watery diarrhoea for two days. He had a significant medical history of well-controlled hypertension. Initial physical examination revealed bilateral lower zone crepitations on auscultation. Laboratory results showed elevated CRP of $63.8 \mathrm{mg} / \mathrm{L}(0.0-5.0 \mathrm{mg} / \mathrm{L})$, lymphopenia of $0.68 \times 10^{9} / \mathrm{L}\left(1.1-3.10 \times 10^{9} / \mathrm{L}\right)$ and elevated lactate dehydrogenase of 796 UL (270-550 UL). Nasopharyngeal RTPCR was positive for SARS-CoV-2. Chest radiograph, obtained on Day 12 of symptoms, revealed ground-glass opacities in the bilateral lower zones (Fig. 2). During admission, elevated direct bilirubin of $67 \mu \mathrm{mol} / \mathrm{L}(1-17 \mu \mathrm{mol} / \mathrm{L})$ and deranged liver enzymes,

${ }^{1}$ Department of Diagnostic Radiology, Tan Tock Seng Hospital, ${ }^{2}$ Department of Radiology, Sengkang General Hospital, ${ }^{3}$ National Centre for Infectious Diseases, ${ }^{4}$ Department of Infectious Diseases, Tan Tock Seng Hospital, ${ }^{5}$ Lee Kong Chian School of Medicine, Nanyang Technological University, Singapore

Correspondence: Dr Tan Cher Heng, Senior Consultant, Department of Diagnostic Radiology, Tan Tock Seng Hospital, 11 Jalan Tan Tock Seng, Singapore 308433. cher_heng_tan@ttsh.com.sg 

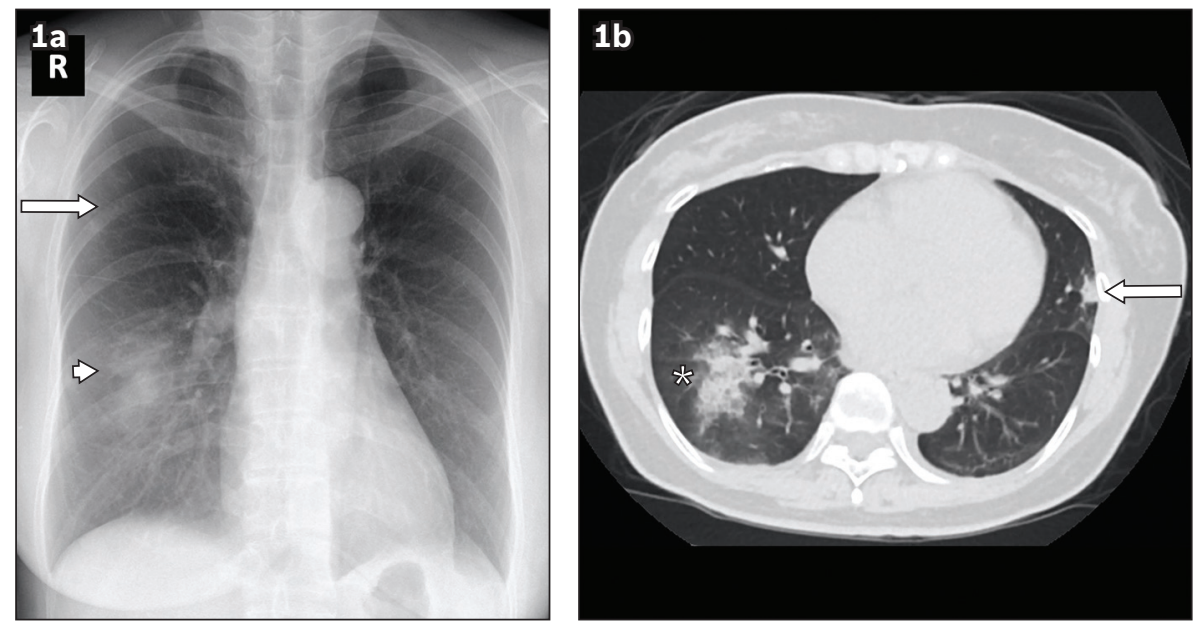

Fig. 1 Case 1: A 52-year-old woman who presented with cough, shortness of breath and fever for four days. (a) Chest radiograph obtained on Day 13 of symptoms shows right lower zone consolidation (arrowhead) and a small focus of ground-glass opacity in the right upper zone (arrow). (b) Axial plain CT image of the lung base obtained on Day 14 of symptoms shows right lower lobe consolidation with surrounding ground-glass opacities (asterisk), a fairly typical appearance in coronavirus disease 2019 pneumonia. A small focus of consolidation is noted in the lingula segment (arrow), which is not discernible on the chest radiograph.
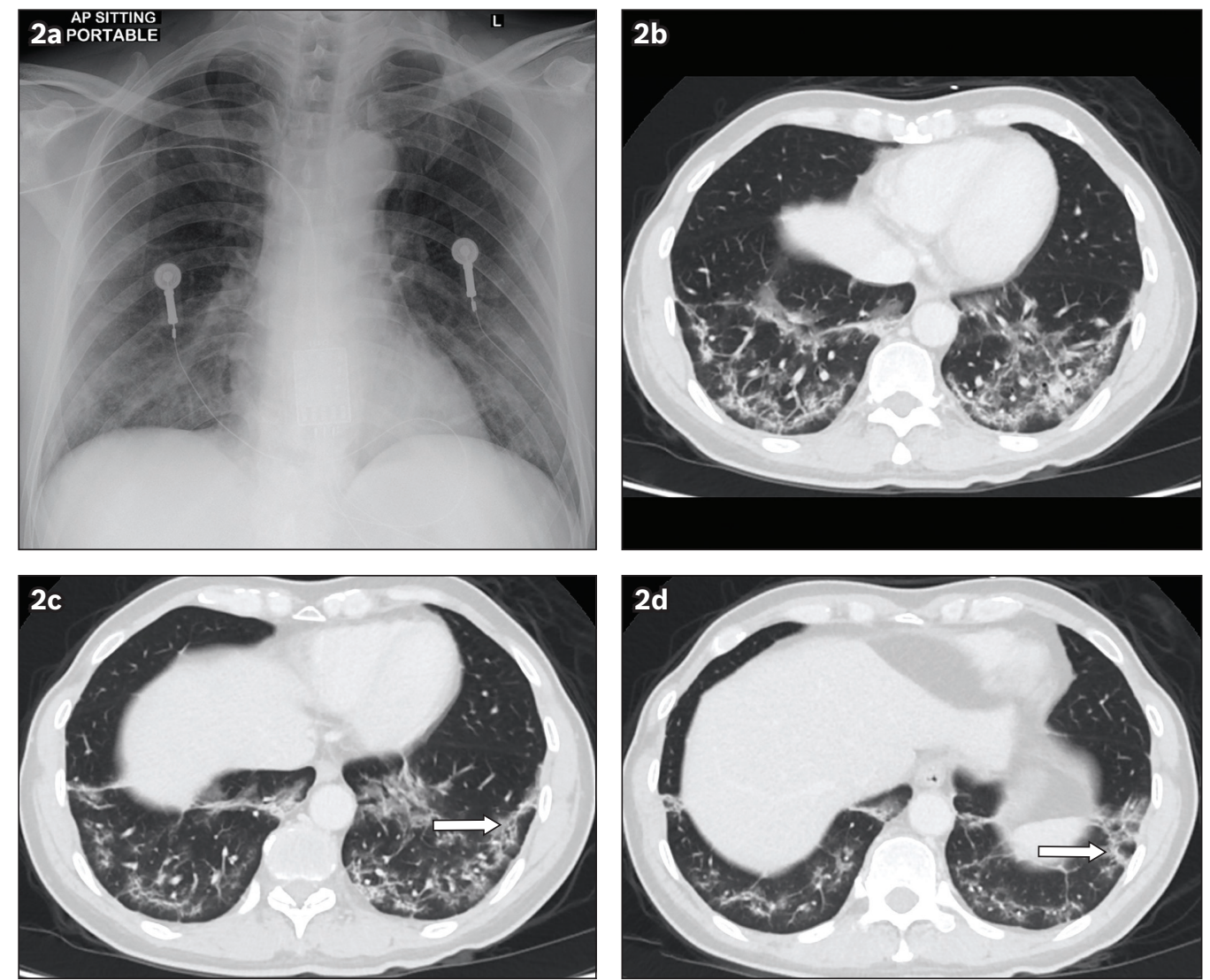

Fig. 2 Case 2: A 56-year-old man who presented with fever and cough for a week. (a) Chest radiograph obtained on Day 12 of symptoms shows groundglass opacities in the bilateral lower lung zones. (b) Axial CT image of the lung base obtained on Day 15 of symptoms shows subpleural reticular bands and ground-glass opacities in the bilateral lower lobes. (c \& d) Perilobular opacities were also appreciated in the left lower lobe (arrows).

in particular gamma-glutamyl transpeptidase of $119 \mathrm{U} / \mathrm{L}$ (9-48 U/L), raised the concern of biliary obstruction. A CT study of the abdomen and pelvis was negative for biliary obstruction. The limited sections of the lung bases demonstrated subpleural reticular bands and ground-glass opacities with perilobular opacities (Fig. 2). The patient remained stable during admission without requiring oxygen supplementation and was discharged well after 12 days.

\section{Case 3}

A 71-year-old man with a history of poorly controlled Type 2 diabetes mellitus presented with fever and lethargy for a week. He had no recent travel or contact history. He was febrile at presentation with a temperature of $38.7^{\circ} \mathrm{C}$ but maintained oxygen saturation of $95 \%$ on room air. Auscultation of the lungs revealed coarse crepitations in the right mid to lower zones and left lower zone. Laboratory results revealed an elevated CRP of $138.2 \mathrm{mg} / \mathrm{L}$ 

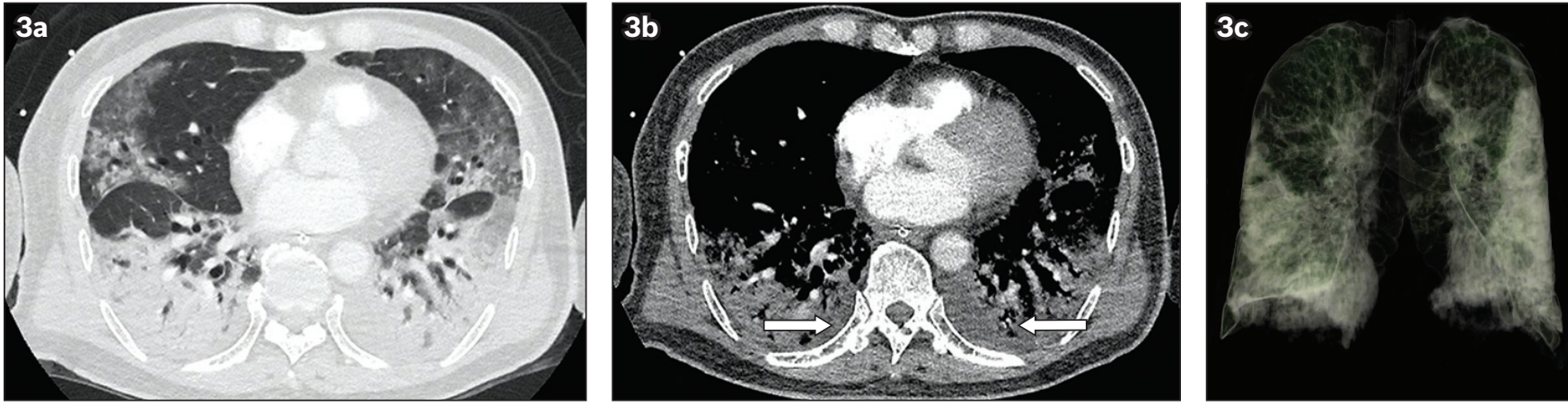

Fig. 3 Case 3: A 71-year-old man who presented with fever and lethargy for a week. CT study was performed on the third week of symptoms. (a) Axial CT images of the lung base in the lung window shows confluent consolidation in the bilateral lower lobes and ground-glass opacities in the bilateral upper lobes with a focal consolidation in the apicoposterior segment of the left upper lobe, layering along the left oblique fissure. (b) Axial CT images of the lung base in the mediastinal window shows bilateral small pleural effusions (arrows), in addition to bilateral lower lobe consolidation. (c) Threedimensional volume CT image of the lungs shows multifocal bilateral lung consolidation involving the central and peripheral locations, significantly worse in the lower lobes.

$(0.0-5.0 \mathrm{mg} / \mathrm{L})$, mildly reduced total white blood cell count of $3.5 \times$ $10^{9} / \mathrm{L}\left(4.0-9.6 \times 10^{9} / \mathrm{L}\right)$ and lymphocyte count of $0.48 \times 10^{9} / \mathrm{L}(1.1-$ $\left.3.1 \times 10^{9} / \mathrm{L}\right)$. Nasopharyngeal RT-PCR was positive for SARS-CoV-2. During admission, he had increasing oxygen supplementation requirement, prompting admission to the intensive care unit, where he was intubated and received mechanical ventilation. CT pulmonary angiography was performed to exclude pulmonary embolism as a cause of desaturation. There was multifocal bilateral lung consolidation with diffuse involvement of the lower lobes (Fig. 3). An anterior-posterior gradient of abnormalities was observed with consolidation affecting the posterior aspects of bilateral lungs, while diffuse ground-glass opacities were noted in the non-dependent areas of the bilateral upper lobes and middle lobe (Fig. 4). Repeat CT pulmonary angiography, performed eight days later, showed regression of ground-glass opacities, which were replaced by organising consolidation and reticular changes (Fig. 5). Bronchiolar dilatation was notable in the lower lobes and bilateral pleural effusions had grown larger. Both studies were negative for pulmonary embolism. Unfortunately, during the admission, he developed right upper limb deep venous thrombosis secondary to prior central venous catheter insertion. This was further complicated by acute lower gastrointestinal bleeding secondary to the use of anticoagulants. CT mesenteric angiography was performed to identify the site of bleeding, which was negative. The limited sections of the lung base showed improvement of bilateral lower lobe consolidation with development of subpleural reticular opacities and traction bronchiectasis related to fibrosis (Fig. 6). The patient remains warded at the time of writing.

\section{DISCUSSION}

A diverse pattern of CT findings in COVID-19 pneumonia has been reported; ground-glass opacities and mixed ground-glass opacities with consolidation are more commonly seen, while other features such as crazy-paving pattern, reverse halo sign, subpleural bands and bronchiectasis have also been described. ${ }^{(4-8)}$ Multifocal bilateral lung involvement, predominantly in the lower lobes and peripheral distribution, was the most common pattern of distribution seen in COVID-19 pneumonia. ${ }^{(4-8)}$ This pattern of multifocal bilateral lower lobe involvement was seen in all
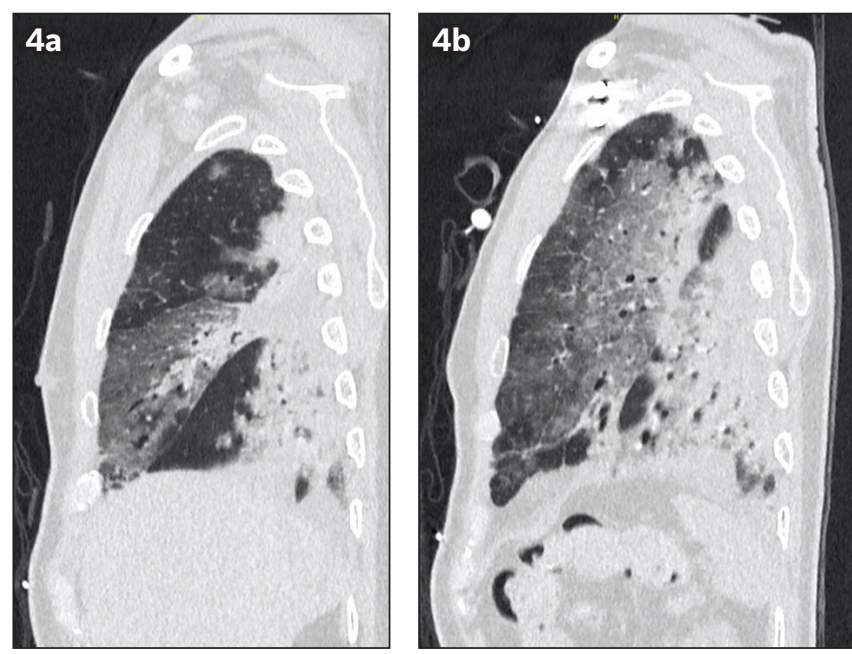

Fig. 4 Case 3: Contrast-enhanced CT images of the (a) right and (b) left lungs in sagittal reconstruction. Chest CT study, obtained on the third week of symptoms, shows anterior-posterior gradient of abnormalities with consolidation affecting the posterior aspects of the bilateral lungs with confluent consolidation seen in both lower lobes and the posterior aspect of the middle lobe. Diffuse ground-glass opacities were noted in the non-dependent areas of the bilateral upper lobes and middle lobe.

three cases in our series. Studies on the evolution of CT findings along the course of the disease showed progression of groundglass opacities with bilateral lung involvement during the first week of symptoms, which worsens into dense consolidation in the second week. ${ }^{(9,10)}$ Following the second week, as the disease improves, there would be resolution of lung consolidation and development of a reticular pattern, septal thickening and bronchiolectasis, suggestive of fibrosis. ${ }^{(9,10)}$ Patchy ground-glass opacities with subpleural bands in bilateral lower lobes, as seen in Case 2 (Fig. 2), likely represent partial convalescence of lung consolidation with early fibrosis. In addition, perilobular opacities were appreciated in this case, a sign described in organising pneumonia. ${ }^{(11)}$ The evolution of lung changes is best illustrated in Case 3, where dense bilateral lung consolidation showed interval resolution and subsequent formation of subpleural reticular opacities and bands with traction bronchiolectasis, indicative of fibrosis. Two recent studies described peak lung involvement about 6-11 days from symptom onset. ${ }^{(12)}$ 

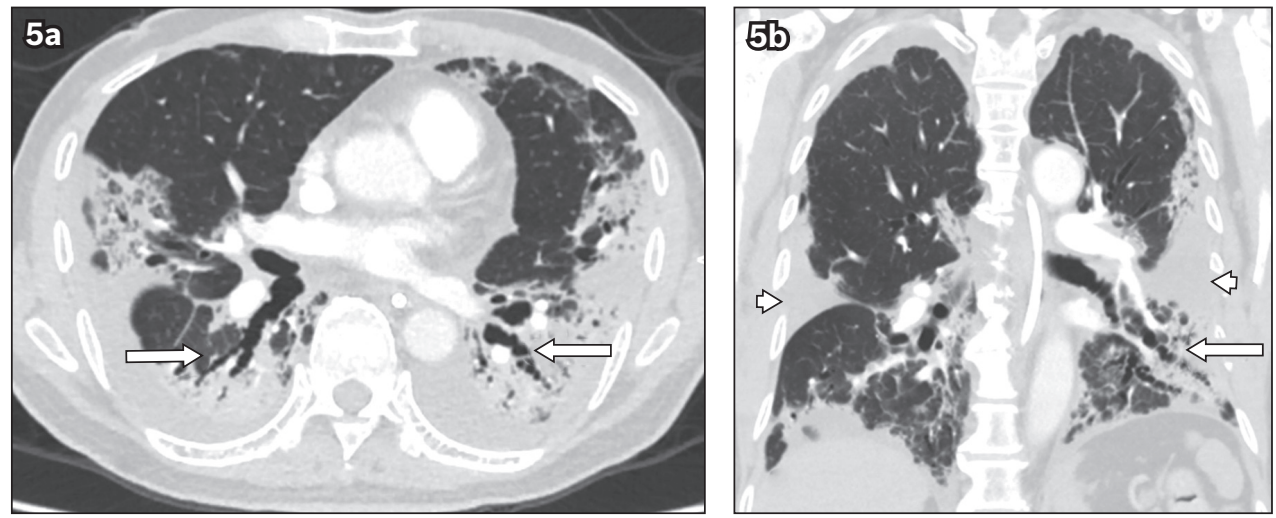

Fig. 5 Case 3: Repeat CT images of the thorax (pulmonary angiography protocol) in (a) axial and (b) coronal planes, performed eight days later, show regression of ground-glass opacities, replaced by organising consolidation and reticular changes. Bronchiolar dilatation was notable in the lower lobes (arrows). Bilateral pleural effusions had grown larger, extending along the pulmonary fissures (arrowheads). The study was negative for pulmonary embolism.
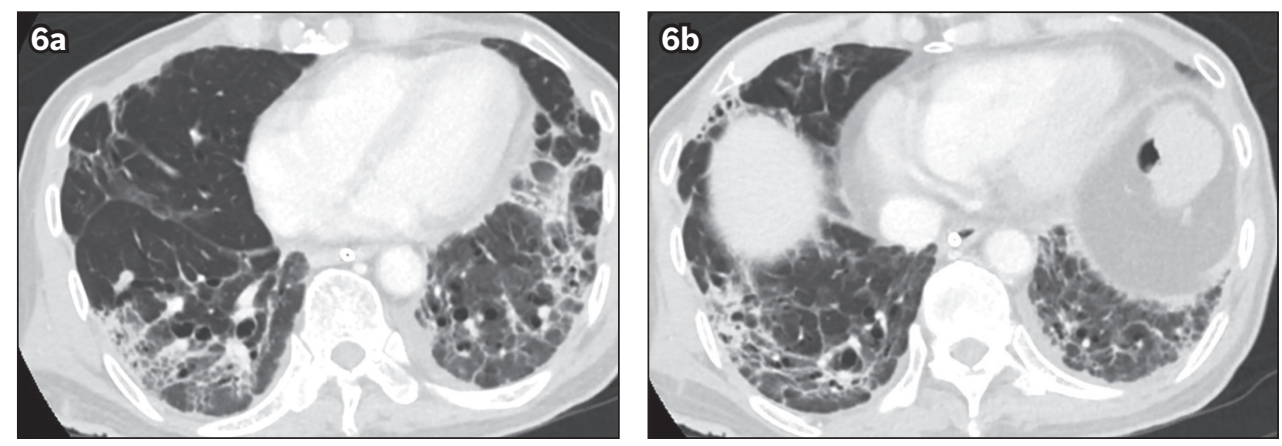

Fig. 6 Case 3: Contrast-enhanced CT images of the lung base, obtained on Week 6 of symptoms (22 days after the initial CT study in Fig. 3). There was improvement of bilateral lower lobe consolidation, with interval development of subpleural reticular opacities and traction bronchiolectasis related to fibrosis. Bilateral pleural effusions have resolved. Disease was predominant in the (a) posterior and (b) basal regions of the lower lobes.

Diffuse lung consolidation, architectural distortion, traction bronchiectasis and pleural effusions have been reported to be more commonly found in severe and fatal disease. ${ }^{(5)}$ These findings were evident in one of our patients (Case 3), who required mechanical ventilation and intensive care support. He developed dense bilateral lung consolidation, patchy ground-glass opacities and bilateral pleural effusions on initial CT study, with follow-up imaging demonstrating development of bronchiolectasis and subpleural bands related to fibrosis. In addition, the presence of an anteriorposterior gradient of lung abnormalities in this patient, with dense consolidation at the dependent regions of the lungs and groundglass opacities in the non-dependent regions, mirrors the classical CT appearance of the acute (wet) phase of acute respiratory distress syndrome (ARDS). ${ }^{(13)}$ In the late phase of ARDS, a coarse reticular pattern and ground-glass opacification, which were deemed to be fine fibrosis beyond the resolution of $\mathrm{CT}$, had been reported in the non-dependent regions of the lungs. ${ }^{(13)}$ However, these findings were not appreciated on the limited sections of the lung base in this patient. Follow-up chest CT studies may identify the long-term effects of COVID-19 pneumonia with ARDS pattern and determine if fibrotic changes of the lungs are reversible.

Hosseiny et al reported significant overlap in the imaging findings of COVID-19 pneumonia with those of severe acute respiratory syndrome (SARS) and Middle East respiratory syndrome (MERS), where peripheral multifocal air space opacities in the form of ground-glass opacities, consolidation or both, were reported. ${ }^{(14)}$ In addition, other viral pneumonias such as adenovirus, herpes simplex virus and cytomegalovirus may present with bilateral lung consolidation. ${ }^{(15)}$ However, at this time during the pandemic, it is highly unlikely for one to be faced with a situation requiring differentiation of these three pathologies in clinical practice. Instead, emphasis should be placed on the recognition of the pattern of lung parenchymal abnormalities that raises the likelihood of the diagnosis of pneumonia, and the subsequent empirical management of the patient based on the travel or contact history. Specifically, this would involve isolation and contact tracing in the effort to contain the spread of disease. Nevertheless, RT-PCR of respiratory samples remains the reference standard for the diagnosis of COVID-19 infection.

CT has been shown to be more sensitive compared to a chest radiograph, being able to detect ground-glass opacities, which are not discernible on a radiograph. ${ }^{(16)}$ In addition, CT provides valuable information on the degree of severity of pneumonia, which aids in the prognostication of the patient. ${ }^{(5)}$ In spite of these advantages, factors such as increased cost, higher radiation dose and the need for longer breath holds for accurate CT image acquisition compared to chest radiographs should also be considered. Furthermore, strict infection control related to the transfer of patients from the isolation room to the radiology department for a CT study and subsequent decontamination of the $\mathrm{CT}$ room following a study needs to be in place.

Several studies have shown that chest CT carries a high sensitivity for the detection of COVID-19 pneumonia..$^{(2,17,18)}$ In a review of 51 patients with COVID-19 pneumonia, the diagnosis 
of viral pneumonia could be made with chest CT three days prior to positive nucleic acid test results. ${ }^{(18)}$ In addition, nucleic acid test has a limited detection rate for SARS-CoV-2, giving rise to false-negative results, and often requires repeated samples. ${ }^{(2,17)}$ In a large retrospective study of 1,014 patients in Wuhan, China, chest CT carried a true-positive rate of $88 \%$ compared to $59 \%$ with RTPCR. ${ }^{(2)}$ In the face of a shortage of PCR test kits and the urgent need for diagnosis despite negative PCR, clinical diagnosis was made based on typical chest CT findings during the peak of the outbreak in Hubei Province, China. ${ }^{(2)}$ This approach may be justifiable in an epidemic area where there is high pre-test probability for the disease, compounded by the limited availability of test kits and laboratory support during the outbreak. However, in our opinion, these results may not be extrapolated to less affected areas with a lower pre-test probability for COVID-19 pneumonia.

In fact, recent recommendations by the American College of Radiology and the British Society of Thoracic Imaging discourage the use of $\mathrm{CT}$ as the first-line diagnostic test to diagnose COVID-19, given its untested specificity. ${ }^{(19,20)}$ In our institution, CT is reserved for severe cases where other causes for desaturation such as pulmonary embolism are suspected, or when there is discordance between the clinical and chest radiographic findings. This explains why the CT appearances of pulmonary involvement for our first two cases were derived from abdominal CT.

In conclusion, this case series of three COVID-19 pneumonia patients highlights the variable chest $\mathrm{CT}$ features during the acute and convalescent phases. Chest CT is a highly sensitive tool for the delineation of the extent of lung disease, but its use as a first-line diagnostic modality to replace RT-PCR is not certain. Nevertheless, CT can define the extent of pulmonary disease for prognostication and associated complications in patients who suffer from more severe disease. Larger-scale studies with longitudinal follow-up would shed light on the evolution of CT findings and the functional sequelae of COVID-19 pneumonia.

\section{REFERENCES}

1. World Health Organization. Coronavirus disease 2019 (COVID-19) Situation Report - 71 Available at: https://www.who.int/docs/default-source/coronaviruse/ situation-reports/20200331-sitrep-71-covid-19.pdf?sfvrsn=4360e92b_6. Accessed April 1, 2020.
2. Ai T, Yang Z, Hou H, et al. Correlation of chest CT and RT-PCR testing in coronavirus disease 2019 (COVID-19) in China: a report of 1014 cases. Radiology 2020 Feb 26. https://doi.org/10.1148/radiol.2020200642. [Epub ahead of print]

3. China National Health Commission. Notice on issuing a new coronavirus infected pneumonia diagnosis and treatment plan (trial version 5). Available at: http://bgs.satcm.gov.cn/zhengcewenjian/2020-02-06/12847.html. Accessed April 1, 2020.

4. Bernheim A, Mei X, Huang M, et al. Chest CT findings in coronavirus disease-19 (COVID-19): relationship to duration of infection. Radiology 2020; 295:200463.

5. Zhao W, Zhong Z, Xie X, Yu Q, Liu J. Relation between chest CT findings and clinical conditions of coronavirus disease (COVID-19) pneumonia: a multicenter study. AJR Am J Roentgenol 2020; 214:1072-7.

6. Zhou S, Wang Y, Zhu T, Xia L. CT features of coronavirus disease 2019 (COVID-19) pneumonia in 62 patients in Wuhan, China. AJR Am J Roentgenol 2020; 214:1287-94.

7. Zu ZY, Jiang MD, Xu PP, et al. Coronavirus disease 2019 (COVID-19): a perspective from China. Radiology 2020 Feb 21. https://doi.org/10.1148/ radiol.2020200490. [Epub ahead of print]

8. Chung M, Bernheim A, Mei X, et al. CT imaging features of 2019 novel coronavirus (2019-nCoV). Radiology 2020; 295:202-7.

9. Pan $F$, Ye $T$, Sun $P$, et al. Time course of lung changes on chest CT during recovery from 2019 novel coronavirus (COVID-19) pneumonia. Radiology 2020; 295:715-21.

10. Shi H, Han X, Jiang N, et al. Radiological findings from 81 patients with COVID-19 pneumonia in Wuhan, China: a descriptive study. Lancet Infect Dis 2020; 20:425-34

11. Ujita M, Renzoni EA, Veeraraghavan S, Wells AU, Hansell DM. Organizing pneumonia: perilobular pattern at thin-section CT. Radiology 2004; 232:757-61.

12. Wang Y, Dong C, Hu Y, et al. Temporal changes of CT findings in 90 patients with COVID-19 pneumonia: a longitudinal study. Radiology 2020 Mar 19. https://doi.org/10.1148/radiol.2020200843. [Epub ahead of print]

13. Sheard S, Rao P, Devaraj A. Imaging of acute respiratory distress syndrome. Respir Care 2012; 57:607-12.

14. Hosseiny M, Kooraki S, Gholamrezanezhad A, Reddy S, Myers L. Radiology perspective of coronavirus disease 2019 (COVID-19): lessons from severe acute respiratory syndrome and Middle East respiratory syndrome. AJR Am J Roentgenol 2020; 214:1078-82

15. Koo HJ, Lim S, Choe J, et al. Radiographic and CT features of viral pneumonia. Radiographics 2018; 38:719-39.

16. Ng MY, Lee EY, Yang J, et al. Imaging profile of the COVID-19 infection: radiologic findings and literature review. Radiol Cardiothorac Imaging 2020 Feb 13. https://doi.org/10.1148/ryct.2020200034. [Epub ahead of print]

17. Dai WC, Zhang HW, Yu J, et al. CT imaging and differential diagnosis of COVID-19. Can Assoc Radiol J 2020; 71:195-200.

18. Li Y, Xia L. Coronavirus disease 2019 (COVID-19): role of chest CT in diagnosis and management. AJR Am J Roentgenol 2020; 214:1280-6.

19. American College of Radiology. ACR recommendations for the use of chest radiography and computed tomography (CT) for suspected COVID-19 infection. Available at: https://www.acr.org/Advocacy-and-Economics/ACR-PositionStatements/Recommendations-for-Chest-Radiography-and-CT-for-SuspectedCOVID19-Infection. Accessed April 1, 2020.

20. British Society of Thoracic Imaging. COVID-19 BSTI statement and guidance. Available at: https://www.bsti.org.uk/standards-clinical-guidelines/clinicalguidelines/covid-19-bsti-statement-and-guidance/. Accessed April 1, 2020. 